Pacific Journal of Mathematics

THE LOCAL STRUCTURE OF SOME MEASURE-ALGEBRA 


\title{
THE LOCAL STRUCTURE OF SOME MEASURE-ALGEBRA HOMOMORPHISMS
}

\section{RUSSELL LYONS}

\begin{abstract}
Extending classical theorems, we obtain representations for bounded linear transformations from $L$-spaces to Banach spaces with a separable predual. In the case of homomorphisms from a convolution measure algebra to a Banach algebra, we obtain a generalization of Šreìder's representation of the Gelfand spectrum via generalized characters. The homomorphisms from the measure algebra on a LCA group, $G$, to that on the circle are analyzed in detail. If the torsion subgroup of $G$ is denumerable, one consequence is the following necessary and sufficient condition that a positive finite Borel measure on $G$ be continuous: $\exists \gamma_{\alpha} \rightarrow \infty$ in $\widehat{G}$ such that $\forall n \neq 0 \quad \hat{\mu}\left(\gamma_{\alpha}^{n}\right) \rightarrow 0$.
\end{abstract}

1. Introduction. Given a measurable space $X$ and a (bounded) complex measure $\mu$ on $X$, the Banach space dual of $L^{1}(\mu)$ is commonly represented as $L^{\infty}(\mu)$. We shall call $M$ an $L$-space on $X$ if $M$ is a Banach space of complex measures on $X$ (under the measure norm) such that $\nu \ll \mu \in M \Rightarrow \nu \in M$ [Sc]. Šreìder [ک̌rr] gave a representation of the dual $M^{*}$ of $M$ as a space of so-called generalized functions, i.e., families of functions $f_{\mu} \in L^{\infty}(\mu)$ satisfying

$$
\begin{gathered}
\nu \ll \mu \Rightarrow f_{\nu}=f_{\mu} \quad \nu \text {-a.e. }, \\
\sup _{\mu \in M}\left\|f_{\mu}\right\|_{L^{\infty}(\mu)}<\infty .
\end{gathered}
$$

The representation of $M^{*}$, like that of $L^{1}(\mu)^{*}$, is by integration:

$$
\mu \mapsto \int f_{\mu} d \mu
$$

Now, given two Banach spaces, $B_{1}$ and $B_{2}$, we denote by $L\left(B_{1}, B_{2}\right)$ the Banach space of bounded linear transformations from $B_{1}$ to $B_{2}$. Since $M^{*}=L(M, \mathrm{C})$, we may ask, in generalizing the above, for a representation of $L(M, B)$, where $B$ is an arbitrary Banach space. Again, the case where $M=L^{1}(\mu)$ is classical [DS]; here, the hypothesis that $B$ has a separable predual is made. In $\S 2$, we extend this theorem to general $L$-spaces $M$ in a manner similar to Sreìder's representation above. In essence, functions are replaced by 
$B$-valued functions. Our treatment will be entirely self contained, thus giving an apparently new proof of [DS, Theorem VI.8.6]. However, another point of view could be adopted. Namely, if we use the Radon-Nikodym theorem to identify $L(\mu)=\{\nu \ll \mu: \nu$ bounded $\}$ with $L^{1}(\mu)$, then we may regard an $L$-space $M$ as the direct limit $\lim _{\mu \in M} L^{1}(\mu)$, where $M$ is directed by $\ll$ and for $\nu \ll \mu, L^{1}(\nu)$ is included in $L^{1}(\mu)$. Now $L(\cdot, B)$ is a functor from the category of Banach spaces to its opposite category and, furthermore, is easily checked to be a left adjoint. Since left adjoints preserve direct limits and inverse limits are dual to direct limits, it follows that $L(M, B)$ is the inverse limit $\lim _{\mu \in M} L\left(L^{1}(\mu), B\right)$, where, for $\nu \ll \mu, L\left(L^{1}(\mu), B\right)$ is mapped by restriction to $L\left(L^{1}(\nu), B\right)$. Hence, given a representation of $L\left(L^{1}(\mu), B\right)$ (as in [DS]) and a construction of inverse limits, we may obtain a representation of $L(M, B)$. This amounts to the same as our Theorem 2.1.

Now Šreìder was actually interested in representing $\Delta M$, the multiplicative linear functionals on $M$, when $M$ was a convolution measure algebra on a locally compact abelian group. He showed that in addition to (1.1) and (1.2), the following property was necessary and sufficient for $f_{\mu}$ to define an element of $\Delta M$ :

$$
\forall \mu, \nu \geq 0 f_{\mu * \nu}(x y)=f_{\mu}(x) f_{\nu}(y) \quad \mu \times \nu \text {-a.e. }[(x, y)] \text {. }
$$

We, too, are mainly interested in the subset of homomorphisms $\operatorname{Hom}(M, B) \subseteq L(M, B)$ when $B$ is a Banach algebra. A similar condition to (1.3) is found in Theorem 3.2. In particular, when $M=M(G)$, the complex Borel measures on a locally compact abelian group, $G$, and $B=M(\mathbf{T}), \mathbf{T}$ the circle, $\operatorname{Hom}(M(G), M(\mathbf{T}))$ contains in a natural way $\operatorname{Hom}(G, \mathbf{T})=\widehat{G}$. The closure of $\widehat{G}$ in a certain weak topology is related to the behavior of Fourier transforms at infinity and contains much information about a measure $\mu$ when regarded locally, i.e., when restricted to $L(\mu)$, or, what is the same, when viewed via the Sreider representation. For example, this analysis will lead to the following surprising result: if the torsion subgroup of $G$ is denumerable, then a positive measure $\mu \in M(G)$ is continuous iff there is a net $\left\{\gamma_{\alpha}\right\} \subseteq \widehat{G}$ tending to infinity such that for all $n \neq 0, \lim _{\alpha} \hat{\mu}\left(\gamma_{\alpha}^{n}\right)=0$. Characterizations of certain other classes of measures are found in $\S 4$; these have proved useful in [KL] and [L4] Other analyses of the local structure of the closure of $\widehat{G}$ for certain $\mu$ can be found in [L3], [L4], and [L5]. The local structure of $\widehat{G}$ is also related to asymptotic distribution; this relationship, described here, has been used in [KL] and [L4]. 
The Šreìder representation, Theorem 3.2, has been given before in [IgK] for the case $\operatorname{Hom}(M, M(\mathrm{~T})), M$ being an $L$-subalgebra of $M(\mathrm{~T})$, though in slightly different notation. An alternative representation for $\operatorname{Hom}(M, M(G))$, where $M$ is a semisimple commutative convolution measure algebra in the sense of Taylor and $G$ is a compact abelian group, analogous to Taylor's representation of $\Delta M$ via a structure semigroup, has been given in [InK].

2. The Šreìder representation of linear transformations. Suppose that $M$ is an $L$-space on a measurable space $X$ and that $B$ is a Banach space with a separable predual, $B_{*}$. Let $\mathscr{B}(X, B)$ denote the set of maps $f: X \rightarrow B$ which are bounded in $B$-norm and measurable when $B$ is given the weak* topology from $B_{*}$. If $f \in \mathscr{B}(X, B)$ and $\mu \in M$, there is a unique element $\int f d \mu \in B$ defined by the relation

$$
\forall b_{*} \in B_{*}\left\langle b_{*}, \int f d \mu\right\rangle=\int_{X}\left\langle b_{*}, f(x)\right\rangle d \mu(x) .
$$

If $D$ is a countable dense set in the unit ball of $B_{*}$, then the equation

$$
\|f(x)\|_{B}=\sup _{b_{*} \in D}\left|\left\langle b_{*}, f(x)\right\rangle\right|
$$

shows that $\|f(\cdot)\|_{B}$ is measurable. It is clear that

$$
\left\|\int f d \mu\right\|_{B} \leq \int\|f\|_{B} d|\mu| .
$$

The set of equivalence classes of $\mathscr{B}(X, B)$ under equality $\mu$-a.e. will be denoted $\mathscr{B}(X, B)_{\mu}$, although this distinction will often be ignored.

The following theorem, which we shall term the Šreider representation, associates to each element of $L(M, B)$ a certain family of maps in $\mathscr{B}(X, B)$. We denote the image of $\mu \in M$ under $\Sigma \in L(M, B)$ by $\Sigma_{\mu}$.

THEOREM 2.1. Let $M$ be an $L$-space and $B$ a Banach space with a separable predual. There is a bijection between $L(M, B)$ and the set of elements $\left\{b_{\cdot, \mu}\right\}_{\mu \in M} \in \prod_{\mu \in M} \mathscr{B}(X, B)_{\mu}$ which satisfy

$$
\sup _{\mu \in M}\|\| b_{x, \mu}\left\|_{B}\right\|_{L^{\infty}(\mu)}<\infty
$$

and

$$
\forall \nu \ll \mu \in M b_{x, \nu}=b_{x, \mu} \quad \nu \text {-a.e. }[x]
$$


such that if $\Sigma$ corresponds to $\{b \cdot, \mu\}_{\mu \in M}$ (written $\left.\Sigma \sim b \cdot, \cdot\right)$, then

$$
\forall \mu \in M \quad \Sigma_{\mu}=\int b_{x, \mu} d \mu(x)
$$

and

$$
\|\Sigma\|_{L(M, B)}=\sup _{\mu \in M}\|\| b_{x, \mu}\left\|_{B}\right\|_{L^{\infty}(\mu)}
$$

Proof. Given $\{b \cdot, \mu\}$ satisfying (i) and (ii), define $\Sigma$ by (iii). If $\mu, \nu \in M$, then by (ii), we have $b_{x, \mu}=b_{x,|\mu|+|\nu|} \mu$-a.e., whence $\Sigma_{\mu}=\int b_{x,|\mu|+|\nu|} d \mu(x)$. In conjunction with similar equations for $\Sigma_{\nu}$ and $\Sigma_{\mu+\nu}$, this equation shows that $\Sigma_{\mu}+\Sigma_{\nu}=\Sigma_{\mu+\nu}$. Similarly, for $\alpha \in \mathbf{C}, \Sigma_{\alpha \mu}=\alpha \Sigma_{\mu}$, whence $\Sigma$ is linear. Let $K$ denote the quantity in (i). Then

$$
\begin{aligned}
\|\Sigma\| & =\sup _{\|\mu\| \leq 1}\left\|\Sigma_{\mu}\right\|=\sup _{\|\mu\| \leq 1}\left\|\int b_{x, \mu} d \mu(x)\right\| \\
& \leq \sup _{\|\mu\| \leq 1} \int\left\|b_{x, \mu}\right\| d|\mu|(x) \leq K .
\end{aligned}
$$

To show that $\|\Sigma\|=K$, choose any nonzero $\mu \in M$ and $\varepsilon>0$. Let $0 \neq \nu \in L(\mu)$ be such that \|\|$b \cdot, \mu\left\|_{B}-\right\|\|b \cdot, \mu\|_{B}\left\|_{L^{\infty}(\mu)}\right\|_{L^{\infty}(\nu)}<\varepsilon$. Let $S$ be the unit sphere of $B$. Since the unit ball of $B$ is weak* compact, there exists a finite number of elements, $b_{*}^{1}, \ldots, b_{*}^{n}$, of the unit ball of $B_{*}$ such that

$$
S=\bigcup_{i=1}^{n}\left\{b \in S:\left|\left\langle b_{*}^{i}, b\right\rangle-1\right|<\varepsilon\right\} .
$$

Therefore $\exists 0<\omega \in L(\nu) \exists i\left\|\left\langle b_{*}^{i}, b_{x, \mu} /\left\|b_{x, \mu}\right\|_{B}\right\rangle-1\right\|_{L^{\infty}(\omega)}<\varepsilon$. We have

$$
\begin{aligned}
\|\Sigma\| & \geq \frac{\left\|\Sigma_{\omega}\right\|}{\|\omega\|} \geq \frac{1}{\|\omega\|}\left|\left\langle b_{*}^{i}, \Sigma_{\omega}\right\rangle\right|=\frac{1}{\|\omega\|}\left|\int\left\langle b_{*}^{i}, b_{x, \mu}\right\rangle d \omega(x)\right| \\
& \geq \frac{1}{\|\omega\|} \int\left\|b_{x, \mu}\right\|_{B} d \omega(x)-\varepsilon K \geq\|\| b \cdot, \mu\left\|_{B}\right\|_{L^{\infty}(\mu)}-\varepsilon(K+1) .
\end{aligned}
$$

Thus $\|\Sigma\|=K$.

Conversely, let $\Sigma \in L(M, B)$. Fix $\mu \in M$. For $b_{*} \in B_{*}$, we denote by $b_{*} \circ \Sigma$ the map $\nu \mapsto\left\langle b_{*}, \Sigma_{\nu}\right\rangle$. Restricted to $L(\mu)$, this map is a bounded linear functional and hence can be represented by a function $g_{b_{*}} \in L^{\infty}(\mu)$. Choose a countable linearly independent set $D$ whose 
linear span over $\mathbf{Q}, D^{\prime}$, is dense in $B_{*}$. If $b_{*}=\sum_{i=1}^{n} \alpha_{i} d_{*}^{i}, d_{*}^{i} \in D$, $\alpha_{i} \in \mathbf{Q}$, define

$$
h_{b_{*}}=\sum_{i=1}^{n} \alpha_{i} g_{d_{*}^{l}} .
$$

Then $b_{*} \mapsto h_{b_{*}}(x)$ is rational-linear on $D^{\prime}$ for every $x \in X$. Furthermore, $h_{b_{*}}=g_{b_{*}} \mu$-a.e., whence by countability of $D^{\prime}$,

$$
\forall b_{*} \in D^{\prime}\left|h_{b_{*}}(x)\right| \leq\left\|b_{*} \circ \Sigma\right\| \leq\left\|b_{*}\right\| \cdot\|\Sigma\|
$$

for $\mu$-a.e. $x$. Now for every $x$ such that $(2.1)$ holds, $b_{*} \mapsto h_{b_{*}}(x)$ extends from $D^{\prime}$ to all of $B_{*}$ as a bounded linear functional, hence element of $B$, call it $f(x)$. This defines $f(x) \mu$-a.e. and shows that, given any $b_{*} \in B_{*}$, if $b_{*}=\lim _{n \rightarrow \infty} b_{*}^{n}\left(b_{*}^{n} \in D^{\prime}\right)$, then

$$
\left\langle b_{*}, f(x)\right\rangle=\lim _{n \rightarrow \infty}\left\langle b_{*}^{n}, f(x)\right\rangle=\lim _{n \rightarrow \infty} h_{b_{*}^{n}}(x)
$$

for every $x$ where $f$ is defined. Write $b ., \mu$ for the equivalence class of $f$. From Equation (2.1), we see that $\|f(x)\| \leq\|\Sigma\|$ for every $x$ where $f$ is defined. Together with (2.2), this shows that $b, \mu \in$ $\mathscr{B}(X, B)_{\mu}$ and gives (i). Now for $\nu \in L(\mu)$ and $b_{*} \in D^{\prime}$, we have

$$
\begin{aligned}
\left\langle b_{*}, \int f d \nu\right\rangle & =\int\left\langle b_{*}, f(x)\right\rangle d \nu(x)=\int h_{b_{*}}(x) d \nu(x) \\
& =\int g_{b_{*}}(x) d \nu(x)=\left\langle b_{*}, \Sigma_{\nu}\right\rangle .
\end{aligned}
$$

Since $D^{\prime}$ is dense, (iii) follows. We claim that $b \cdot, \mu$ is uniquely determined by the property just established:

$$
\forall \nu \in L(\mu) \Sigma_{\nu}=\int b_{x, \mu} d \nu(x) .
$$

Indeed, if we also have that $\forall \nu \in L(\mu) \Sigma_{\nu}=\int b_{x, \mu}^{\prime} d \nu(x)$ for some $b^{\prime}, \mu \in \mathscr{B}(X, B)_{\mu}$, then

$$
\forall b_{*} \in D^{\prime} \forall \nu \in L(\mu) \int\left\langle b_{*}, b_{x, \mu}\right\rangle d \nu(x)=\int\left\langle b_{*}, b_{x, \mu}^{\prime}\right\rangle d \nu(x),
$$

whence for $\mu$-a.e. $x \forall b_{*} \in D^{\prime}\left\langle b_{*}, b_{x, \mu}\right\rangle=\left\langle b_{*}, b_{x, \mu}^{\prime}\right\rangle$, i.e., $b_{x, \mu}=$ $b_{x, \mu}^{\prime} \mu$-a.e. Thus (ii) follows. The same argument shows that if $\Sigma \sim$ $b_{.,}$. and $\Sigma \sim b_{\cdot, .}^{\prime}$, then $b_{., .}=b_{\cdot, .}^{\prime}$.

We define the weak* operator topology ( $\left.\mathrm{W}^{*} \mathrm{OT}\right)$ on $L(M, B)$ as the weakest topology such that $\forall \mu \in M \quad \forall b_{*} \in B_{*} \Sigma \mapsto\left\langle b_{*}, \Sigma_{\mu}\right\rangle$ is continuous. It is an elementary exercise to show that the unit ball of $L(M, B)$ is $\mathrm{W}^{*} \mathrm{OT}$ compact. 
For $\mu \in M$, let $L(M, B)_{\mu}$ denote the set of Šreìder representations $b_{,, \mu}$ of elements of $L(M, B)$. We give $L(M, B)_{\mu}$ the weak topology generated by the maps $b ., \mu \mapsto \int\left\langle b_{*}, b_{x, \nu}\right\rangle d \nu(x) \quad\left(b_{*} \in B_{*}\right.$, $\nu \in L(\mu))$. Thus, the $\mathrm{W}^{*} \mathrm{OT}$ is the inverse limit of these topologies, i.e., it is the weak topology generated by the maps $\Sigma \mapsto b \cdot, \mu \quad(\mu \in M)$ from $L(M, B) \rightarrow L(M, B)_{\mu}$, where $\Sigma \sim b .,$.

Every decomposition $M=I \oplus J$ of $M$ as a direct sum of closed subspaces yields an addition on $L(M, B)$ as follows: if $\Pi^{1}, \Pi^{2} \in$ $L(M, B)$, then we may define

$$
\Sigma_{\mu}=\Pi_{\mu_{I}}^{1}+\Pi_{\mu_{J}}^{2}
$$

where $\mu=\mu_{I}+\mu_{J}, \mu_{I} \in I, \mu_{J} \in J$. If $\Sigma \sim b_{., .}, \Pi^{i} \sim b_{., .}^{i}$, and $I \perp J$, then $b_{x, \mu}=b_{x, \mu_{I}}^{1}+b_{x, \mu_{J}}^{2} \mu$-a.e.

The case where $B=M(Y)$, the space of complex regular Borel measures on a locally compact metric space, $Y$, is of interest. A predual of $B$ is the separable space $C_{0}(Y)$ of continuous functions vanishing at infinity. We shall denote the Sreider representation of $\Sigma$ by $\sigma_{x, \mu}$ in this case; thus, if $f \in C_{0}(Y)$ and $\mu \in M$,

$$
\int_{Y} f d \Sigma_{\mu}=\int_{X}\left(\int_{Y} f d \sigma_{x, \mu}\right) d \mu(x) .
$$

(If $Y$ is separable and a countable union of complete subspaces, then (2.4) holds for $f \in \mathscr{B}(Y, \mathbf{C})$ since it is preserved under bounded pointwise limits. In particular, for Borel sets $E \subseteq Y$,

$$
\left.\Sigma_{\mu}(E)=\int_{X} \sigma_{x, \mu}(E) d \mu(x) .\right)
$$

Let $M^{+}$denote the nonnegative elements of $M$ and likewise for $M^{+}(Y)$. We say that $\Sigma \in L(M, M(Y))$ is positive if it carries $M^{+}$ into $M^{+}(Y)$. It is easy to see from (2.4) applied to $|\mu|$ that $\Sigma \geq 0$ iff $\forall \mu \in M \quad \forall^{e} x[\mu] \sigma_{x, \mu} \geq 0$ (" $\forall e x[\mu]$ " means "for $\mu$-a.e. $x$ "-see [L1]). It is also easy to show that if $\Sigma \geq 0$, then $\nu \ll \mu \Rightarrow \Sigma_{\nu} \ll \Sigma_{|\mu|}$ and $\left|\Sigma_{\mu}\right| \leq \Sigma_{|\mu|}$.

3. The Šreìder representation of homomorphisms. Let $G$ be a locally compact semigroup with separately continuous multiplication. Then $M(G)$ is a Banach algebra under convolution [W]. Let $M$ be an $L$ subalgebra of $M(G)$, i.e., a subalgebra which is also an $L$-subspace, and let $B$ be a Banach algebra with a separable predual such that 
multiplication is separately weak* measurable and

$$
\begin{aligned}
& \forall f \in \mathscr{B}(G, B) \forall b \in B \forall \mu \in M \\
& \qquad f(x) \cdot b d \mu(x)=\left(\int f d \mu\right) \cdot b \\
& \qquad \& \int b \cdot f(x) d \mu(x)=b \cdot \int f d \mu .
\end{aligned}
$$

In order to state some sufficient conditions that (3.1) be true, we define the following multiplication on $B^{*} \times B$. If $b \in B$ and $b^{*} \in B^{*}$, then $b^{\prime} \mapsto\left\langle b^{\prime} \cdot b, b^{*}\right\rangle$ is a bounded linear functional on $B$; we denote it by $b^{*} \cdot b$. Let $\bar{B}_{*}^{s w^{*}}$ be the smallest subspace of $B^{*}$ containing (canonically) $B_{*}$ which is closed under weak* sequential limits. Let $\Delta B$ be the subset of $B^{*}$ consisting of the multiplicative linear functionals.

Proposition 3.1. Let $B$ be a Banach algebra with a separable predual. Right multiplication on $B$ is weak* measurable and the first equation of (3.1) holds if any of the following conditions is satisfies:

(i) $B_{*} \cdot B \subseteq \bar{B}_{*}^{s w^{*}}$.

(ii) Right multiplication is weak* continuous.

(iii) Right multiplication is weak ${ }^{*}$ measurable and $\bar{B}_{*}^{s w^{*}} \cap \Delta B$ separates points in $B$.

Proof. The class of $b^{*} \in B^{*}$ such that $b \mapsto\left\langle b, b^{*}\right\rangle$ is weak* measurable contains $B_{*}$ and is closed under weak* sequential limits. Thus, all elements of $\bar{B}_{*}^{s w^{*}}$ are weak* measurable. Now right multiplication is weak* measurable iff $\forall b \in B \quad \forall b_{*} \in B_{*} \quad b^{\prime} \mapsto\left\langle b_{*}, b^{\prime} \cdot b\right\rangle$ is weak* measurable. But $\left\langle b_{*}, b^{\prime} \cdot b\right\rangle=\left\langle b^{\prime}, b_{*} \cdot b\right\rangle$, whence this condition is equivalent to all elements of $B_{*} \cdot B$ being weak* measurable. The sufficiency of (i) for measurability is now obvious. Also, the class of weak* measurable $b^{*} \in B^{*}$ such that

$$
\left\langle\int f d \mu, b^{*}\right\rangle=\int\left\langle f, b^{*}\right\rangle d \mu
$$

is closed under weak* sequential limits by the bounded convergence theorem, hence contains $\bar{B}_{*}^{s w^{*}}$. Thus, if (i) holds, then $\forall b_{*} \in B_{*}$ $\forall b \in B$

$$
\begin{aligned}
\left\langle b_{*}, \int f \cdot b d \mu\right\rangle & =\int\left\langle b_{*}, f \cdot b\right\rangle d \mu=\int\left\langle f, b_{*} \cdot b\right\rangle d \mu \\
& =\left\langle\int f d \mu, b_{*} \cdot b\right\rangle=\left\langle b_{*},\left(\int f d \mu\right) \cdot b\right\rangle,
\end{aligned}
$$

whence the first equation of (3.1). 
Now (ii) is equivalent to $B_{*} \cdot B \subseteq B_{*}$ since $B_{*}$ is the set of weak* continuous linear functionals on $B$. Thus, sufficiency follows from that of (i). Finally, if (iii) holds, then for $f \in \mathscr{B}(G, B), b \in B$, $\mu \in M$, and $b^{*} \in \bar{B}_{*}^{s w^{*}} \cap \Delta B$, we have

$$
\begin{gathered}
\left\langle\int f \cdot b d \mu, b^{*}\right\rangle=\int\left\langle f \cdot b, b^{*}\right\rangle d \mu=\int\left\langle f, b^{*}\right\rangle\left\langle b, b^{*}\right\rangle d \mu \\
=\int\left\langle f, b^{*}\right\rangle d \mu \cdot\left\langle b, b^{*}\right\rangle=\left\langle\int f d \mu, b^{*}\right\rangle \cdot\left\langle b, b^{*}\right\rangle \\
=\left\langle\left(\int f d \mu\right) \cdot b, b^{*}\right\rangle,
\end{gathered}
$$

from which the first equation of (3.1) follows.

Let $\mathscr{B}_{0}(G, B)$ denote the Baire-measurable functions from $G$ to $B$, where $B$ is given the weak* topology. For $\mu, \nu \in M(G)$, let $\mu \times \nu$ denote, besides the usual product measure, also its unique extension to a regular Borel measure in $M(G \times G)$. If $f \in \mathscr{B}_{0}(G, B)$ and $\mu, \nu \in M(G)$, then

$$
\begin{aligned}
\int f d \mu * \nu & =\int f(x y) d \mu \times \nu(x, y) \\
& =\iint f(x y) d \mu(x) d \nu(y)
\end{aligned}
$$

as can be seen by applying any $b_{*} \in B_{*}[\mathrm{~W}]$.

The Šreìder representation of $\operatorname{Hom}(M, B)$, the continuous homomorphisms from $M$ to $B$, satisfies one property additional to those in Theorem 2.1 .

THEOREM 3.2. Let $G$ be a locally compact semigroup with separately continuous multiplication and $M$ an L-subalgebra of $M(G)$. Let $B$ be a Banach algebra with a separable predual and separately weak*. measurable multiplication satisfying (3.1). Let $\Sigma \in L(M, B)$ and choose $b_{., \mu} \in \mathscr{B}_{0}(G, B) \quad(\mu \in M)$ so that $\Sigma \sim b_{., .}$Then $\Sigma \in$ $\operatorname{Hom}(M, B)$ iff

$$
\forall \mu, \nu \in M^{+} b_{x y, \mu * \nu}=b_{x, \mu} \cdot b_{y, \nu} \text { for } \mu \times \nu \text {-a.e. }(x, y)
$$


Proof. Suppose first that (3.2) is satisfied. Then for $\mu, \nu \in M$,

$$
\begin{aligned}
\Sigma_{\mu * \nu} & =\int b_{t,|\mu| *|\nu|} d \mu * \nu(t)=\iint b_{x y,|\mu| *|\nu|} d \mu(x) d \nu(y) \\
& =\iint b_{x,|\mu|} \cdot b_{y,|\nu|} d \mu(x) d \nu(y) \\
& =\int\left(\int b_{x,|\mu|} d \mu(x)\right) \cdot b_{y,|\nu|} d \nu(y) \\
& =\int b_{x,|\mu|} d \mu(x) \cdot \int b_{y,|\nu|} d \nu(y)=\Sigma_{\mu} \cdot \Sigma_{\nu} .
\end{aligned}
$$

Conversely, if $\Sigma \in \operatorname{Hom}(M, B)$, then given $\mu, \nu \in M^{+}$, we have for all $\mu^{\prime} \in L(\mu)$ and $\nu^{\prime} \in L(\nu)$,

$$
\begin{aligned}
\int b_{x y, \mu * \nu} d \mu^{\prime} \times \nu^{\prime}(x, y)=\int b_{t, \mu * \nu} d \mu^{\prime} * \nu^{\prime}(t)=\Sigma_{\mu^{\prime} * \nu^{\prime}} \\
=\Sigma_{\mu^{\prime}} \cdot \Sigma_{\nu^{\prime}}=\int b_{x, \mu} d \mu^{\prime}(x) \cdot \int b_{y, \nu} d \nu^{\prime}(y) \\
=\iint b_{x, \mu} \cdot b_{y, \nu} d \mu^{\prime}(x) d \nu^{\prime}(y) \\
=\int b_{x, \mu} \cdot b_{y, \nu} d \mu^{\prime} \times \nu^{\prime}(x, y) .
\end{aligned}
$$

Since the span of $L(\mu) \times L(\nu)$ is dense in $L(\mu \times \nu)$, (3.2) follows.

If multiplication in $B$ is jointly weak* continuous (for example, if $B_{*} \cap \Delta B$ separates points in $\left.B\right)$, then the unit ball in $\operatorname{Hom}(M, B)$ is easily shown to be $\mathrm{W}^{*} \mathrm{OT}$ compact. An example where compactness fails is $\operatorname{Hom}(M(\mathbf{R}), M(\mathbf{R}))$ : define $T_{n}(n \geq 1)$ in the unit ball by

$$
\int_{\mathbf{R}} f(x) d\left(T_{n}\right)_{\mu}(x)=\int_{\mathbf{R}} f(n x) d \mu(x) \quad\left(f \in C_{0}(\mathbf{R})\right)
$$

and let $\Sigma: \mu \mapsto \mu(\{0\}) \delta(0)$, where $\delta(0)$ is the Dirac measure at 0 . Then $T_{n} \rightarrow \Sigma$ in $\mathrm{W}^{*} \mathrm{OT}$, but

$$
\Sigma \in L(M(\mathbf{R}), M(\mathbf{R})) \backslash \operatorname{Hom}(M(\mathbf{R}), M(\mathbf{R})) .
$$

We define the following multiplication on $L(M, B):$ if $\Sigma \sim b_{.}$, and $\Pi \sim b_{.}, .$, then $\Sigma \cdot \Pi$ is defined by its Sreider representation $b_{x, \mu} \cdot b_{x, \mu}^{\prime}$. When $B$ is commutative, $\operatorname{Hom}(M, B)$ is closed under multiplication. It is easily verified that if multiplication in $B$ is separately weak* continuous, then multiplication in $L(M, B)$ is separately $\mathrm{W}^{*} \mathrm{OT}$ continuous. 
Suppose that $M=I \oplus J$, where $I$ is a closed ideal and $J$ is a closed subalgebra. If $\Pi^{1}, \Pi^{2} \in \operatorname{Hom}(M, B)$ satisfy

$$
\forall \mu \in I \forall \nu \in J \Pi_{\mu * \nu}^{1}=\Pi_{\mu}^{1} \cdot \Pi_{\nu}^{2} \quad \& \quad \Pi_{\nu * \mu}^{1}=\Pi_{\mu}^{2} \cdot \Pi_{\mu}^{1},
$$

then the "sum" $\Sigma$ of $\Pi^{1}$ and $\Pi^{2}$ defined in (2.3) is a homomorphism.

4. Limit points of group homomorphisms. If $H$ is a locally compact group, then convolution is separately weak* continuous in $M(H)$. Indeed, if $\mu_{\alpha}, \mu, \nu \in M(H)$ with $\mu_{\alpha} \stackrel{w^{*}}{\longrightarrow} \mu$, then for $f \in C_{0}(H)$, the map $x \mapsto \int f(x y) d \nu(y)$ lies in $C_{0}(H)$, whence

$$
\begin{aligned}
\int f d \mu_{\alpha} * \nu & =\iint f(x y) d \nu(y) d \mu_{\alpha}(x) \\
& \rightarrow \iint f(x y) d \nu(y) d \mu(x)=\int f d \mu * \nu,
\end{aligned}
$$

which is to say that $\mu_{\alpha} * \nu \stackrel{w^{*}}{\longrightarrow} \mu * \nu$. A similar argument applies to $\nu * \mu_{\alpha}$. Thus, if $G$ is a locally compact semigroup with separately continuous multiplication and $H$ is a locally compact metrizable group, then the preceding section applied to $\operatorname{Hom}(M, M(H))$ for any $L$-subalgebra $M$ of $M(G)$. Every continuous homomorphism $\varphi: G \rightarrow H$ yields an element of $\operatorname{Hom}(M, M(H))$, which we also denote by $\varphi$, defined by $\left\langle f, \varphi_{\mu}\right\rangle=\langle f \circ \varphi, \mu\rangle$ for $f \in C_{0}(H)$. The Sreider representation of such a $\varphi$ is particularly simple: $\varphi \sim \delta(\varphi(x))$ (independent of $\mu$ ), where $\delta(t)$ denotes the Dirac measure at $t$.

We identify $\operatorname{Hom}(G, H)$ with a subset of $\operatorname{Hom}(M(G), M(H))$ in the above manner. Our aim is to study the set

$$
\Lambda=\overline{\operatorname{Hom}(G, H)} \backslash \operatorname{Hom}(G, H)
$$

and its local structure

$$
\Lambda(\mu)=\left\{\Sigma_{\mu}: \Sigma \in \Lambda\right\}, \quad \breve{\Lambda}(\mu)=\left\{\sigma_{\cdot, \mu}: \sigma_{.,} \in \widetilde{\Lambda}\right\},
$$

where $\breve{\Lambda}$ consists of the Šreider representations of elements of $\Lambda$. Since all elements of $\operatorname{Hom}(G, H)$ are positive and lie in the unit ball, the same holds for $\Lambda$. (In fact, every positive homomorphism lies in the unit ball: if $0 \leq \Sigma \in \operatorname{Hom}(M(G), M(H))$, then for $\mu \in M(G)$ and $n \geq 1$, we have

$$
\left\|\Sigma_{\mu}\right\|^{n} \leq\left\|\Sigma_{|\mu|}\right\|^{n}=\left\|\Sigma_{|\mu|}^{n}\right\|=\left\|\Sigma_{|\mu|^{n}}\right\| \leq\|\Sigma\| \cdot\left\|\left.\mu\right|^{n}\right\|=\|\Sigma\| \cdot\|\mu\|^{n},
$$

whence $\|\Sigma\| \leq 1$.)

We are particularly interested in the case where $G$ is a locally compact abelian group and $H$ is a circle group, $\mathbf{T}$. In this case, 
$\operatorname{Hom}(G, \mathbf{T})=\widehat{G}$, the dual of $G$, and the identification of $\widehat{G}$ as a subset of $\operatorname{Hom}(M(G), M(T))$ preserves the usual topology of $\widehat{G}$ (of uniform convergence on compact subsets). Furthermore, as $\widehat{G}$ lies in the unit ball of $\operatorname{Hom}(M(G), M(\mathbf{T}))$, it follows that $\overline{\widehat{G}}=\widehat{G} \cup \Lambda$ is a compactification of $\widehat{G}$.

Recall that a sequence $\left\{x_{k}\right\}_{k=1}^{\infty} \subseteq G$ is said to have an asymptotic distribution $\sigma$, written $\left\{x_{k}\right\} \sim \sigma$, if

$$
\frac{1}{K} \sum_{k=1}^{K} \delta\left(x_{k}\right) \stackrel{w^{*}}{\longrightarrow} \sigma \quad \text { as } K \rightarrow \infty .
$$

For $n \in \mathbf{Z}$ and $\Sigma \in \operatorname{Hom}(M(G), M(\mathbf{T}))$, define $\widehat{\boldsymbol{\Sigma}}(n) \in \Delta M(G)$ by $\langle\mu, \widehat{\Sigma}(n)\rangle=\widehat{\boldsymbol{\Sigma}}_{\mu}(n)$. We write the Šreider representation of $\chi \in$ $\Delta M(G)$ as $\chi_{\mu}(x)$. Thus, if $\Sigma \sim \sigma_{.}$, and $\chi=\widehat{\Sigma}(n)$, then

$$
\chi_{\mu}(x)=\hat{\sigma}_{x, \mu}(n) \text {. }
$$

Note that for all $n$, the map $\Sigma \mapsto \widehat{\Sigma}(n)$ from $(\operatorname{Hom}(M(G), M(T))$, $\mathrm{W}^{*} \mathrm{OT}$ ) to $\Delta M(G)$ (with its usual Gelfand topology) is continuous. We regard the Fourier transform as a restriction of the Gelfand transform; thus, in accordance with the Šreider representation, we have $\hat{\mu}(\gamma)=\int \gamma d \mu$ for $\gamma \in \widehat{G}$.

PROPOSITION 4.1. Let $G$ be a locally compact abelian group and $\Lambda=\overline{\widehat{G}} \backslash \widehat{G}$ in $\operatorname{Hom}(M(G), M(T))$. Then

(i) $\Lambda$ is closed topologically and under multiplication by elements of $\overline{\widehat{G}}$;

(ii) if $\sigma_{x}, \tau_{x} \in \breve{\Lambda}(\mu)$, then $\sigma_{x} * \tau_{x} \in \breve{\Lambda}(\mu)$;

(iii) $\Lambda(\mu)=\left\{\nu \in M(\mathbf{T}): \exists\right.$ net $\left\{\gamma_{\alpha}\right\} \subseteq \widehat{G} \quad\left(\gamma_{\alpha} \rightarrow \infty \& \forall n \in \mathbf{Z}\right.$ $\left.\left.\hat{\mu}\left(\gamma_{\alpha}^{n}\right) \rightarrow \hat{\nu}(n)\right)\right\}$;

(iv) $\breve{\Lambda}(\mu)=\left\{\sigma . \in \mathscr{B}(G, M(\mathbf{T}))_{\mu}: \exists\right.$ net $\left\{\gamma_{\alpha}\right\} \subseteq \widehat{G}\left(\gamma_{\alpha} \rightarrow \infty \& \forall n \in\right.$ $\mathbf{Z} \gamma_{\alpha}^{n} \rightarrow \hat{\sigma} .(n)$ weak* in $\left.\left.L^{\infty}(\mu)\right)\right\}$;

(v) if $G$ is metrizable, then the nets in (iii) and (iv) can be replaced by sequences and $\breve{\Lambda}(\mu)=\left\{\sigma . \in \mathscr{B}(G, M(\mathbf{T}))_{\mu}: \exists \gamma_{j} \in \widehat{G}\right.$ $\left(\gamma_{j} \rightarrow \infty \&\right.$ for every subsequence $\left.\left.\gamma_{j_{k}}, \forall^{e} x[\mu]\left\{\gamma_{j_{k}}(x)\right\}_{k=1}^{\infty} \sim \sigma_{x}\right)\right\}$.

Proof. Suppose that $\Sigma \in \Lambda$ is the limit of a net $\left\{\gamma_{\alpha}\right\} \subseteq \widehat{G}$. Then $\widehat{\boldsymbol{\Sigma}}(n)=\lim \gamma_{\alpha}^{n}$ in $\Delta M(G)$ for all $n \in \mathbf{Z}$. Now if $\gamma_{\alpha} \rightarrow \gamma \in \widehat{G}$, then $\gamma_{\alpha}^{n} \rightarrow \gamma^{n}$, whence $\Sigma=\gamma$. But since $\Lambda \cap \widehat{G}=\varnothing$, this is impossible, and so $\gamma_{\alpha} \rightarrow \infty$ in $\widehat{G}$. In particular, $\widehat{\Sigma}(1)$ is 0 on $L^{1}(G)$ [HMP, 
p. 136, Proposition 4] and consequently $\Lambda$ is closed. It is clear that $\Lambda \cdot \widehat{G} \subseteq \Lambda$, from which (i) now follows. Statement (ii) ensues as well. Now if $\nu \in \Lambda(\mu)$, then let $\widehat{G} \ni \gamma_{\alpha} \rightarrow \Sigma \in \Lambda$ be such that $\nu=\Sigma_{\mu}$. Then $\gamma_{\alpha} \rightarrow \infty$ and $\left(\gamma_{\alpha}\right)_{\mu} \stackrel{w^{*}}{\longrightarrow} \Sigma_{\mu}=\nu$, which gives the inclusion $\subseteq$ of (iii). On the other hand, if $\gamma_{\alpha} \rightarrow \infty$ and $\forall n \hat{\mu}\left(\gamma_{\alpha}^{n}\right) \rightarrow \hat{\nu}(n)$, then by compactness of $\overline{\widehat{G}}$, we can choose a subnet $\left\{\gamma_{\beta}^{\prime}\right\}$ of $\left\{\gamma_{\alpha}\right\}$ converging to some $\Sigma$. Since $\gamma_{\beta}^{\prime} \rightarrow \infty$, it follows that $\Sigma \in \Lambda$ and $\nu=\Sigma_{\mu} \in \Lambda(\mu)$. This completes the proof of (iii). The proof of (iv) is analogous. Finally, if $G$ is metrizable, then $L^{1}(\mu)$ is separable for $\mu \in M(G)$ and so $L(M(G), M(\mathbf{T}))_{\mu}$ is metrizable. Thus, if $\mu \in M(G)$ and $\gamma_{\alpha} \rightarrow \Sigma \sim \sigma .,$. , pick any non-zero $\rho \in L^{1}(G)$ and a subsequence $\left\{\delta\left(\gamma_{\alpha_{j}}(\cdot)\right)\right\}$ converging to $\sigma_{\cdot,|\mu|+|\rho|}$ in $L(M(G), M(\mathbf{T}))_{|\mu|+|\rho|}$. Then $\gamma_{\alpha_{J}}=\delta\left(\gamma_{\alpha_{j}}(\cdot)\right)^{\wedge}(1) \stackrel{w^{*}}{\longrightarrow}(\widehat{\Sigma}(1))_{\rho}=0$ in $L^{\infty}(\rho)$, whence $\gamma_{\alpha_{J}} \rightarrow \infty$ in $\widehat{G}$, and $\gamma_{\alpha_{j}}^{n} \stackrel{w^{*}}{\longrightarrow}(\widehat{\Sigma}(n))_{\mu}=\hat{\sigma}_{\cdot, \mu}(n)$ in $L^{\infty}(\mu)$. This shows the sufficiency of sequences for (iii) and (iv). Furthermore, if $\forall n \gamma_{j}^{n} \rightarrow$ $\hat{\sigma} .(n)$ weak* in $L^{\infty}(\mu)$, then by [L2, Lemma 5], there is a subsequence $\left\{\gamma_{j}^{\prime}\right\}$ of $\left\{\gamma_{j}\right\}$ such that every further subsequence $\left\{\gamma_{j_{k}}^{\prime}\right\}$ satisfies

$$
\forall^{e} x[\mu]\left\{\gamma_{j_{k}}^{\prime}(x)\right\}_{k=1}^{\infty} \sim \sigma_{x} .
$$

Conversely, if $\left\{\gamma_{j}\right\}$ is a sequence, every subsequence of which satisfies

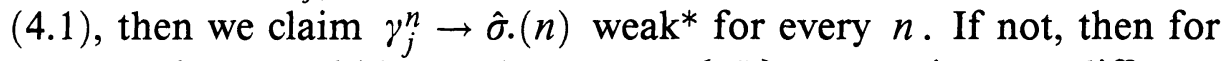
some $n$ there would be a subsequence $\left\{\gamma_{j_{k}}^{n}\right\}$ converging to a different limit $\chi$. Then also

$$
\frac{1}{K} \sum_{k=1}^{K} \gamma_{j_{k}}^{n} \stackrel{w^{*}}{\longrightarrow} \chi
$$

and by (4.1),

$$
\frac{1}{K} \sum_{k=1}^{K} \gamma_{j_{k}}^{n} \stackrel{w^{*}}{\longrightarrow} \hat{\sigma} .(n) .
$$

Therefore $\chi=\hat{\sigma} .(n)$, a contradiction. Thus (v) follows from (iv).

When $\widehat{G}$ is regarded as a subset of $\Delta M(G)$, we shall use the notation $\Gamma$ rather than $\widehat{G}$ to avoid confusion. Let $T_{n} \in \operatorname{Hom}(G, G)$ denote the map $x \mapsto x^{n} \quad(n \in \mathbf{Z})$, as well as the corresponding map induced in $\operatorname{Hom}(M(G), M(G))$. Thus, for $\Sigma \in \operatorname{Hom}(M(G), M(\mathbf{T}))$, we obtain $\Sigma \circ T_{n} \in \operatorname{Hom}(M(G), M(\mathbf{T}))$; note that if $\Sigma=\gamma \in \widehat{G}$, then $\gamma \circ T_{n}=\gamma^{n}$. 
Proposition 4.2. Let $G$ be a LCA group and

$$
\Sigma \in \operatorname{Hom}(M(G), M(T)) .
$$

Then $\Sigma \in \overline{\widehat{G}}$ iff $\widehat{\Sigma}(1) \in \bar{\Gamma}$ and $\forall n \in \mathbf{Z} \widehat{\boldsymbol{\Sigma}}(n)=\widehat{\boldsymbol{\Sigma}}(1) \circ T_{n}$. The map $\Sigma \mapsto \widehat{\Sigma}(1)$ is an isomorphism from $\overline{\widehat{G}}$ onto $\bar{\Gamma}$ sending $\widehat{G}$ to $\Gamma$.

Proof. If $\Sigma \in \overline{\widehat{G}}$, let $\widehat{G} \ni \gamma_{\alpha} \stackrel{\mathrm{W}^{*} \mathrm{OT}}{\longrightarrow} \Sigma$. Since $\hat{\gamma}_{\alpha}(n)=\gamma_{\alpha}^{n}$, we have $\gamma_{\alpha}^{n} \rightarrow \widehat{\boldsymbol{\Sigma}}(n)$ for all $n$. In particular, $\widehat{\boldsymbol{\Sigma}}(1) \in \bar{\Gamma}$. Also, $\widehat{\boldsymbol{\Sigma}}(n)=\lim \gamma_{\alpha}^{n}=$ $\lim \gamma_{\alpha} \circ T_{n}=\left(\lim \gamma_{\alpha}\right) \circ T_{n}=\widehat{\Sigma}(1) \circ T_{n}$. Conversely, if $\widehat{\Sigma}(1) \in \bar{\Gamma}$ and $\forall n \widehat{\Sigma}(n)=\widehat{\Sigma}(1) \circ T_{n}$, then let $\gamma_{\alpha} \rightarrow \widehat{\Sigma}(1)$. Choose a convergent subnet $\gamma_{\beta}^{\prime} \rightarrow \Pi$ in $\operatorname{Hom}(M(G), M(T))$. Then from the above, $\widehat{\Pi}(n)=\widehat{\Pi}(1) \circ$ $T_{n}=\widehat{\Sigma}(1) \circ T_{n}=\widehat{\Sigma}(n)$ for all $n$, whence $\Sigma=\Pi \in \overline{\widehat{G}}$.

It follows from this that the map $\Sigma \mapsto \widehat{\Sigma}(1)$ is injective. Surjectivity onto $\bar{\Gamma}$ is proved by a compactness argument similar to the above.

We write $M(G)=M_{c}(G) \oplus M_{d}(G)$ for the decomposition of a measure into its continuous and discrete parts. Then $h_{d}: \mu \mapsto \int_{G} d \mu_{d}=$ $\hat{\mu}_{d}(0)$ is in $\bar{\Gamma} \backslash \Gamma$ [HMP, pp. 136-7, (4.1.4)]. We denote the element of $\Lambda$ corresponding to $h_{d}$ by $\Pi^{d}$. If $G$ has at most countably many torsion elements, then we claim that

$$
\widehat{\Pi}^{d}(n)= \begin{cases}0 & \text { if } n=0, \\ h_{d} & \text { if } n \neq 0,\end{cases}
$$

whence

$$
\Pi_{\mu}^{d}=\hat{\mu}_{c}(0) \lambda+\hat{\mu}_{d}(0) \delta(0),
$$

where $\lambda$ is Lebesgue measure on $\mathbf{T}$. To see this, note first that

$$
\widehat{\Pi}^{d}(0): \mu \mapsto\left(\mu \circ T_{0}^{-1}\right)^{\wedge}(0)=\hat{\mu}(0) .
$$

Second, if $n \neq 0$, then for all $g \in G$, there are, by the supposition, denumerably many $x \in G$ such that $x^{n}=g$. Therefore

$$
\left(\mu \circ T_{n}^{-1}\right)(\{g\})=\sum_{x^{n}=g} \mu(\{x\}),
$$

whence

$$
\begin{aligned}
\widehat{\Pi}^{d}(n): \mu & \mapsto \sum_{g \in G}\left(\mu \circ T_{n}^{-1}\right)(\{g\}) \\
& =\sum_{g \in G} \sum_{x^{n}=g} \mu(\{x\})=\sum_{x \in G} \mu(\{x\})=\hat{\mu}_{d}(0) .
\end{aligned}
$$

This proves the claim. 
Related elements of $\Lambda$ are $\Sigma \cdot \Pi^{d}$ for $\Sigma \in \overline{\widehat{G}}$; if, as above, the torsion subgroup of $G$ is denumerable, then

$$
\left(\Sigma \cdot \Pi^{d}\right)_{\mu}=\hat{\mu}_{c}(0) \lambda+\Sigma_{\mu_{d}} \text {. }
$$

Thus, if we set $\Pi: \mu \mapsto \hat{\mu}(0) \lambda$, then $\Sigma \cdot \Pi^{d}$ is the sum of $\Pi$ and $\Sigma$ defined by (2.3) and (3.3) from the decomposition $M=M_{c} \oplus M_{d}$. An interesting example is $G=\mathbf{T}$ and $\Sigma: \mu \mapsto \mu$; in this case, $\left(\Sigma \cdot \Pi^{d}\right)_{\mu}=$ $\hat{\mu}_{c}(0) \lambda+\mu_{d}$.

Provided still that $G$ has a denumerable torsion subgroup, the Šreìder representation $\pi_{\text {.,. }}^{d}$ of $\Pi^{d}$ is given by

$$
\pi_{x, \mu}^{d}= \begin{cases}\lambda & \text { if } \mu(\{x\})=0, \\ \delta(0) & \text { if } \mu(\{x\}) \neq 0 .\end{cases}
$$

Let $\lambda \in \mathscr{B}(G, M(\mathbf{T}))_{\mu}$ be defined by $\lambda(x) \equiv \lambda$. Then from [HMP, p. 70, Corollaire 2] and Proposition 4.2 (or from (4.2) and the following proposition),

$$
\mu \in M_{c}(G) \Leftrightarrow \lambda \in \breve{\Lambda}(\mu) .
$$

This yields other characterizations of $M_{c}(G)$ when combined with Proposition 4.1 (iv), (v). For example,

$$
\begin{aligned}
\mu \in M_{c}(G) & \Leftrightarrow \exists \gamma_{\alpha} \rightarrow \infty \forall \nu \in L(\mu) \forall n \neq 0 \hat{\nu}\left(\gamma_{\alpha}^{n}\right) \rightarrow 0 \\
& \Leftrightarrow \exists \gamma_{\alpha} \rightarrow \infty \forall \gamma \in \widehat{G} \forall n \neq 0 \hat{\mu}\left(\gamma \gamma_{\alpha}^{n}\right) \rightarrow 0 .
\end{aligned}
$$

Our next proposition describes $\breve{\Lambda}(\mu)$ completely when $\mu$ is discrete (cf. [HMP, pp. 67-68]).

Proposition 4.3. Let $G$ be a LCA group. Let $\overline{\widehat{G}}$ denote the Sreider representations of $\overline{\widehat{G}} \subseteq \operatorname{Hom}(M(G), M(\mathbf{T}))$ and, for $\mu \in$ $M(G), \overline{\widehat{G}}(\mu)=\left\{\sigma_{,}, \mu: \sigma_{.,} \in \in \overline{\overline{\widehat{G}}}\right\}$. Let $G_{d}$ denote $G$ with the discrete topology and, for $\mu \in M_{d}(G)$, let $G_{d}^{\mu}$ denote the discrete subgroup generated by the mass-points of $\mu$.

(i) $\forall \Sigma \in \overline{\widehat{G}} \exists \varphi \in \widehat{G}_{d} \forall \mu \in M_{d}(G) \quad \Sigma_{\mu}=\sum_{x \in G} \mu(\{x\}) \delta(\varphi(x))$ and $\sigma_{x, \mu}=\delta(\varphi(x))$, where $\Sigma \sim \sigma .,$.

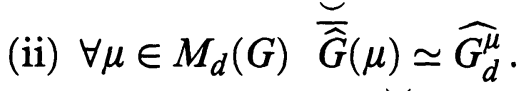

(iii) $\mu \in M_{d}(G) \Leftrightarrow \overline{\widehat{G}}(\mu)$ is a group (under the multiplication in $L(M(G), M(\mathrm{~T})))$. 
Proof. (i) Let $\widehat{G} \ni \gamma_{\alpha} \stackrel{\mathrm{W}^{*} \mathrm{OT}}{\longrightarrow} \Sigma$. Then for $x \in G$,

$$
\delta\left(\gamma_{\alpha}(\cdot)\right) \rightarrow \sigma_{\cdot, \delta(x)} \in L(M(G), M(\mathbf{T}))_{\delta(x)},
$$

i.e., $\delta\left(\gamma_{\alpha}(x)\right)=\sigma_{x, \delta(x)}$ eventually. Thus, $\gamma_{\alpha}(x)$ stabilizes at some point $\varphi(x)$ and $\sigma_{x, \delta(x)}=\delta(\varphi(x))$. The assertions now follow from linearity and properties of the Šreider representation.

(ii) The fact that $\breve{\widehat{G}}(\mu)$ can be identified as a compact subgroup of $\widehat{G}_{d}^{\mu}$ follows from (i). If it were not the whole group, then there would be a nonzero $x \in G_{d}^{\mu}$ such that $\varphi(x)=1$ for all $\varphi \in \overline{\overline{\widehat{G}}}(\mu)$. In particular, $\gamma(x)=1$ for all $\gamma \in \widehat{G}$, whence $x=0$, a contradiction.

(iii) This follows from [HMP, p. 68, Proposition 10] and (ii).

We now arrive at the characterization of positive continuous measures mentioned in the introduction.

THEOREM 4.4. Let $G$ be a LCA group whose torsion subgroup is denumerable and let $\mu \in M^{+}(G)$ be positive. Then $\mu \in M_{c}^{+}(G)$ iff there is a net $\widehat{G} \ni \gamma_{\alpha} \rightarrow \infty$ such that for all $n \neq 0, \hat{\mu}\left(\gamma_{\alpha}^{n}\right) \rightarrow 0$.

Proof. By Proposition 4.1 (iii), this is equivalent to $\mu \in M_{c}^{+}(G) \Leftrightarrow$ $\hat{\mu}(0) \lambda \in \Lambda(\mu)$. For $\mu \in M_{c}^{+}(G)$, this follows from $\lambda \in \breve{\Lambda}(\mu)$ (see (4.3)). If $\mu \notin M_{c}^{+}(G)$ and $\Sigma \in \Lambda$, then $\Sigma_{\mu}=\Sigma_{\mu_{c}}+\Sigma_{\mu_{d}} \geq \Sigma_{\mu_{d}}$ since $\mu_{c} \geq 0$ and $\Sigma \geq 0$. However, by Proposition 4.3(i), $\Sigma_{\mu_{d}}$ is nonzero and discrete; hence $\Sigma_{\mu}$ cannot equal $\hat{\mu}(0) \lambda$.

Because of the interest this theorem may present, we provide the following "elementary" proof and strengthening for the case $G=\mathbf{T}$. If $\mu \in M_{c}(T)$, then by Wiener's theorem [K, p. 42], there is a sequence $\left\{m_{k}^{(1)}\right\}$ of density one in $\mathbf{N}$ such that $\hat{\mu}\left(m_{k}^{(1)}\right) \rightarrow 0$. Likewise, there is a sequence $\left\{m_{k}^{(n)}\right\}$ of density one such that $\hat{\mu}\left(n m_{k}^{(n)}\right)=\left(\widehat{\left.T_{n}\right)_{\mu}}\left(m_{k}^{(n)}\right) \rightarrow\right.$ 0 since $\left(T_{n}\right)_{\mu} \in M_{c}$, for $n \neq 0$. By an elementary intersection argument, we obtain a sequence $\left\{m_{k}\right\}$, still of density one, such that for all $n \neq 0, \hat{\mu}\left(n m_{k}\right) \rightarrow 0$. (A similar argument produces a sequence $\left\{m_{k}\right\}$ of density one such that for $n \neq 0$ and all $r, \hat{\mu}\left(r+n m_{k}\right) \rightarrow 0$, i.e., $\delta\left(m_{k} x\right) \rightarrow \lambda$ in $L(M(\mathbf{T}), M(\mathbf{T}))_{\mu}$, thereby strengthening (4.3).) For the converse, we use the following proof due to Jean-François Méla. Let $K_{l}(x)$ be the Fejér kernel of order $l$. Then if $\mu \geq 0$ and if for 
all $n \neq 0, \hat{\mu}\left(n m_{k}\right) \rightarrow 0$, then

$$
\mu(\{0\}) \leq \int_{\mathrm{T}} \frac{1}{2 l+1} K_{l}\left(m_{k} x\right) d \mu(x) \rightarrow \frac{1}{2 l+1} \hat{\mu}(0) \quad \text { as } k \rightarrow \infty
$$

by hypothesis. Since this is true for all $l$, it follows that $\mu(\{0\})=0$. Now apply this result to $\mu * \tilde{\mu}$, where $\tilde{\mu}(E)=\mu(-E)$.

The local structure of $\Lambda$ can be used to characterize other classes of measures besides $M_{c}$ and $M_{d}$. If $\mathscr{C}$ is a class of subsets of $G$, let

$$
\mathscr{C}^{\perp}=\{\mu \in M(G): \forall E \in \mathscr{C}|\mu|(E)=0\} .
$$

Thus, if $\mathscr{D}$ is the class of singletons, $\mathscr{D}^{\perp}=M_{c}(G)$.

Definition. A set $E \subseteq G$ is called an $H$-set if there is a sequence $\widehat{G} \ni \gamma_{k} \rightarrow \infty$ such that $\left\{\gamma_{k}(x): k \geq 1, x \in E\right\}$ is not dense in T. A set $E \subseteq G$ is called a Dirichlet set if there is a sequence $\widehat{G} \ni \gamma_{k} \rightarrow \infty$ such that $\lim _{k \rightarrow \infty} \sup _{x \in E}\left|\gamma_{k}(x)-1\right|=0$. A measure $\mu \in M(G)$ is called a Dirichlet measure if $\varlimsup_{\lim _{\gamma \rightarrow \infty}}|\widehat{\mu}|(\gamma) \mid=\|\mu\|$.

For background on $H$-sets, see [Z, Chapters IX, XII]; on Dirichlet sets and measures, see [HMP, pp. 34-35, 240-247]. The following proposition is used in [KL].

Proposition 4.5. Let $G$ be a LCA group.

(i) If $G$ is metrizable, then

$$
\begin{aligned}
H^{\perp} & =\left\{\mu: \forall \sigma . \in \breve{\Lambda}(\mu) \forall^{e} x[\mu] \operatorname{supp} \sigma_{x}=\mathbf{T}\right\} \\
& =\left\{\mu: \forall \Sigma \in \Lambda \forall \nu \in L(\mu) \operatorname{supp} \Sigma_{\nu}=\mathbf{T}\right\}
\end{aligned}
$$

(ii) $\mu$ is a Dirichlet measure iff the constant function $\boldsymbol{\delta}(\mathbf{0}) \in \Lambda(\mu)$. (iii) $D^{\perp}=\left\{\mu: \forall \sigma . \in \breve{\Lambda}(\mu) \forall e x[\mu] \sigma_{x} \neq \delta(0)\right\}$

Proof. Part (i) follows from Proposition 4.1(v) and a straightforward generalization of [L4, Theorem 13]. Part (ii) follows from Proposition 4.2 and the fact that $\mu$ is a Dirichlet measure iff the constant function $\mathbf{1} \in(\bar{\Gamma} \backslash \Gamma)(\mu)$ [HMP, p. 34, Lemma 6]. Part (iii) follows from part (ii) and the fact that $D^{\perp}$ consists of the measures orthogonal to the Dirichlet measures [HMP, p. 243, Proposition 9].

Our final remarks concern the circle group.

Definition. A positive measure $\mu \in M^{+}(\mathbf{T})$ is called $C$-quasisymmetric if for all pairs of adjacent arcs, $I$ and $J$, on $\mathbf{T}$ of equal 
length, $\mu I \leq C \cdot \mu J$. We denote the class of $C$-quasisymmetric measures by $Q S(C)$.

Note that quasisymmetric measures are continuous.

Proposition 4.6. The class $Q S(C)$ is weak ${ }^{*}$ closed. If $\mu \in Q S(C)$, then $\Lambda(\mu) \subseteq Q S(C), \breve{\Lambda}(\mu) \subseteq Q S(C)$ in the sense that if $\sigma . \in \overleftarrow{\Lambda}(\mu)$, then $\forall^{e} x[\mu] \quad \sigma_{x} \in Q S(C)$, and $\Lambda(\nu) \subseteq Q S(C)$ for all $0 \leq \nu \in L(\mu)$.

Proof. Let $Q S(C) \ni \mu_{\alpha} \stackrel{w^{*}}{\longrightarrow} \nu$. Given adjacent arcs $I, J$ of equal length and $\varepsilon>0$, pick $f, g \in C(\mathbf{T})$ such that $f \leq \mathbf{1}_{I}, \mathbf{1}_{J} \leq g$, $\int\left(\mathbf{1}_{I}-f\right) d \nu \leq \varepsilon$, and $\int\left(g-\mathbf{1}_{J}\right) d \nu \leq \varepsilon$. We have

$$
\begin{aligned}
\nu I & \leq \int f d \nu+\varepsilon=\lim \int f d \mu_{\alpha}+\varepsilon \leq \varlimsup \lim \mu_{\alpha} I+\varepsilon \\
& \leq C \cdot \varlimsup \lim \mu_{\alpha} J+\varepsilon \leq C \cdot \lim \int g d \mu_{\alpha}+\varepsilon=C \int g d \nu+\varepsilon \\
& \leq C \cdot \nu J+(C+1) \varepsilon .
\end{aligned}
$$

Since $\varepsilon$ was arbitrary, we see that $\nu I \leq C \cdot \nu J$, whence $\nu \in Q S(C)$.

Choose $\mu \in Q S(C)$. Then $\gamma_{\mu} \in Q S(C)$ for any $\gamma \in \widehat{\mathbf{T}}$. Since $\Lambda(\mu)$ is contained in the weak* closure of $\left\{\gamma_{\mu}\right\}_{\gamma \in \widehat{\mathrm{T}}}$, it follows that $\Lambda(\mu) \subseteq Q S(C)$. Suppose that $E \subseteq \mathbf{T}$ and $\mu E>0$. If $I$ and $J$ are adjacent arcs of equal length and $\varepsilon>0$, then choose $U$, a finite union of arcs, such that $\mu(U \triangle E) \leq \varepsilon$. By continuity of $\mu$, we have for all large $\gamma$,

$$
\begin{aligned}
\mu\left(E \cap \gamma^{-1}[I]\right) & \leq \mu\left(U \cap \gamma^{-1}[I]\right)+\varepsilon \leq C \cdot \mu\left(U \cap \gamma^{-1}[J]\right)+2 \varepsilon \\
& \leq C \cdot \mu\left(E \cap \gamma^{-1}[J]\right)+(C+2) \varepsilon .
\end{aligned}
$$

Since $\varepsilon$ was arbitrary, it follows that $\Lambda\left(\left.\mu\right|_{E}\right) \subseteq Q S(C)$. As $Q S(C)$ is a positive cone, we deduce that $\Lambda(\nu) \subseteq Q S(C)$ for $0 \leq \nu \in L(\mu)$. Finally, let $\sigma . \in \breve{\Lambda}(\mu)$. Let $P$ be the essential range of $\sigma$., i.e, the smallest weak* closed set $P$ such that $\sigma_{x} \in P \mu$-a.e. Then $P$ is contained in the weak* closure of $\left\{\int \sigma_{x} d \nu(x): 0 \leq \nu \in L(\mu),\|\nu\|=1\right\}=$ $\bigcup\{\Lambda(\nu): 0 \leq \nu \in L(\mu),\|\nu\|=1\}$, which, by the above, is contained in $Q S(C)$.

As an example of the pathology possible for $\Lambda(\mu)$, we present the following observation.

Proposition 4.7. There is a measure $\mu \in M(\mathbf{T})$ such that for any probabilitv measure $\nu \in M(\mathbf{T})$, there exists $\sigma . \in \breve{\Lambda}(\mu)$ such that $\sigma_{x}=$ $\nu \mu$-a.e. 
Proof. Let $\left\{P_{k}\right\}_{k \geq 1}$ be a set of trigonometric polynomials such that $\left\{P_{k} \cdot \lambda\right\}$ is weak* dense in the set of probability measures. Let $\left\{n_{k}\right\} \subseteq$ $\mathbf{N}$ satisfy $n_{k+1} \geq 3 n_{k} \cdot \operatorname{deg} P_{k}$. Form the generalized Riesz product [HMP, Chapitre 5] $\mu=\prod_{k \geq 1} P_{k}\left(n_{k} x\right)$. Then given a probability $\nu$, let $P_{k_{l}} \lambda \stackrel{w^{*}}{\longrightarrow} \nu$. For any $r, m \in \mathbf{Z}$, it is easy to see that $\hat{\mu}\left(r+m n_{k_{l}}\right) \rightarrow$ $\hat{\mu}(r) \hat{\nu}(m)$, i.e., $\delta\left(n_{k_{1}} x\right) \rightarrow \nu$ in $L(M(\mathbf{T}), M(\mathbf{T}))_{\mu}$.

\section{REFERENCES}

[DS] N. Dunford and J. T. Schwartz, Linear Operators. Part I: General Theory. Wiley, New York, 1958, p. 503.

[HMP] B. Host, J.-F. Méla, and F. Parreau, Analyse Harmonique des Mesures, Astérisque, 135-136 (1986).

[IgK] S. Igari and Y. Kanjin, Homomorphisms of measure algebras on the unit circle, J. Math. Soc. Japan, 31 (1979), 503-512.

[InK] J. Inoue and Y. Kanjin, Homomorphisms of convolution measures algebras and convolution semigroups of measures, Hokkaido Math. J., 10 (1981) Sp., 285-302.

[KL] A. S. Kechris and R. Lyons, Ordinal rankings on measures annihilating thin sets, Trans. Amer. Math. Soc., 310 (1988), 747-758.

[L1] R. Lyons, Measure-theoretic quantifiers and Haar measure, Proc. Amer. Math. Soc., 86 (1982), 67-70; Erratum, Proc. Amer. Math. Soc., 91 (1984), 329-330.

[L2] _ Fourier-Stieltjes coefficients and asymptotic distribution modulo 1, Ann. of Math., 122 (1985), 155-170.

[L3] _ The size of some classes of thin sets, Studia Math., 86 (1987), 59-78.

[L4] _ Mixing and asymptotic distribution modulo 1, Ergodic Theory Dynamical Systems, 8 (1988), 597-619.

[L5] _ Singular measures with spectral gaps, Proc. Amer. Math. Soc., 310 (1988), 747-758.

[Sc] H. H. Schaefer, Banach Lattices and Positive Operators, Springer, Berlin, 1974.

[ั̌r] Y. A. Šreider, The structure of maximal ideals in rings of measures with involution, Mat. Sb. (N.S), 27 (69) (1950), 297-318; Amer. Math. Soc. Transl. (1st ser.) No. 81 (1953).

[W] J. C. S. Wong, Convolution and separate continuity, Pacific J. Math., 75 (1978), 601-611.

[Z] A. Zygmund, Trigonometric Series. 2nd ed., reprinted. Volumes I, II. Cambridge University Press, Cambridge, 1979.

Received August 15, 1988 and in revised form December 6, 1989. Partially supported by an AMS Research Fellowship and an NSF Postdoctoral Fellowship.

STANFORD UNIVERSITY

STANFORD, CA 94305-2125

Current address: Indiana University

Bloomington, IN 47405 


\section{PACIFIC JOURNAL OF MATHEMATICS EDITORS}

\author{
V. S. VARADARAJAN \\ (Managing Editor) \\ University of California \\ Los Angeles, CA 90024-1555-05 \\ Herbert Clemens \\ University of Utah \\ Salt Lake City, UT 84112 \\ THOMAS ENRIGHT \\ University of California, San Diego \\ La Jolla, CA 92093
}

R. FINN

Stanford University

Stanford, CA 94305

HeRmanN FlaschKa

University of Arizona

Tucson, AZ 85721

VAUGHAN F. R. JONES

University of California

Berkeley, CA 94720

STEVEN KERCKHOFF

Stanford University

Stanford, CA 94305
C. C. MOORE

University of California

Berkeley, CA 94720

Martin ScharlemanN

University of California

Santa Barbara, CA 93106

\section{HAROLD STARK}

University of California, San Diego La Jolla, CA 92093

\section{ASSOCIATE EDITORS}
R. ARENS
E. F. BECKENBACH
B. H. NeumanN
F. WolF
K. YoshidA
(1906-1982)

(1904-1989)
TIONS

UNIVERSITY OF ARIZONA

UNIVERSITY OF BRITISH COLUMBIA

CALIFORNIA INSTITUTE OF TECHNOLOGY

UNIVERSITY OF CALIFORNIA

MONTANA STATE UNIVERSITY

UNIVERSITY OF NEVADA, RENO

NEW MEXICO STATE UNIVERSITY

OREGON STATE UNIVERSITY
UNIVERSITY OF OREGON

UNIVERSITY OF SOUTHERN CALIFORNIA

STANFORD UNIVERSITY

UNIVERSITY OF HAWAII

UNIVERSITY OF TOKYO

UNIVERSITY OF UTAH

WASHINGTON STATE UNIVERSITY

UNIVERSITY OF WASHINGTON 


\section{Pacific Journal of Mathematics}

\section{Vol. 148, No. $1 \quad$ March, 1991}

David Marion Arnold and Charles Irvin Vinsonhaler, Duality and

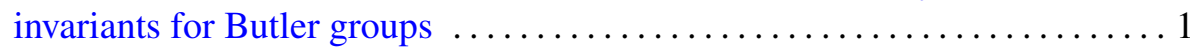

Philippe Delanoë, Obstruction to prescribed positive Ricci curvature . . . . . 11

María J. Druetta, Nonpositively curved homogeneous spaces of dimension

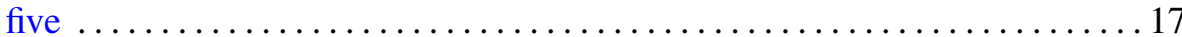

Robert Fitzgerald, Combinatorial techniques and abstract Witt rings III .... 39

Maria Girardi, Dentability, trees, and Dunford-Pettis operators on $L_{1} \ldots \ldots 59$

Krzysztof Jarosz, Ultraproducts and small bound perturbations $\ldots \ldots \ldots \ldots 81$

Russell David Lyons, The local structure of some measure-algebra homomorphisms .................................. 89

Fiona Anne Murnaghan, Asymptotic behaviour of supercuspidal characters of $p$-adic $\mathrm{GL}_{3}$ and $\mathrm{GL}_{4}$ : the generic unramified case $\ldots \ldots \ldots \ldots \ldots 107$

H. Rouhani, Quasi-rotation $C^{*}$-algebras $\ldots \ldots \ldots \ldots \ldots \ldots \ldots \ldots \ldots \ldots \ldots \ldots \ldots \ldots$

Ignacio Sols Lucía, Michał Szurek and Jaroslaw Wisniewski, Rank-2

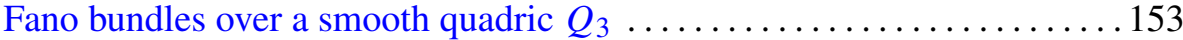

Martin Strake and Gerard Walschap, Ricci curvature and volume

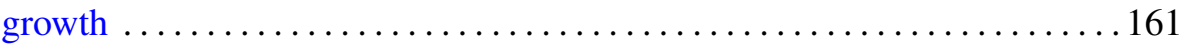

Anton Ströh and Johan Swart, A Riesz theory in von Neumann algebras . . 169

Ming Wang, The classification of flat compact complete space-forms with metric of signature $(2,2)$ 